\title{
Facial gangrenous ecthyma: case report
}

\begin{abstract}
Gangrenous Ecthyma is an infrequent infection caused by Gram negative bacteria, mainly Pseudomona aeruginosa. We describe the clinical case of a patient with active oncohematologic disease who had an infection with rhinosinusal origin, with Pseudomona aeruginosa bacteraemia and required antibiotic therapy; extensive resections of facial necrotic tissue and left orbital exenteration in order to cure the infection.
\end{abstract}

Keywords: gangrenous ecthyma, rhinosinusal, necrotizing fasciitis
Volume 10 Issue $6-2018$

\author{
Carlos Santiago Ruggeri,' Ana Laura Cajelli, ${ }^{2}$ \\ Ana Inés Legris, ${ }^{3}$ Rodrigo Molachino ${ }^{3}$ \\ 'ENT Department, Hospital Italiano de Buenos Aires, Argentina \\ ${ }^{2}$ Rhinosinusology Fellowship, ENT Department, Hospital Italiano \\ de Buenos Aires, Argentina \\ ${ }^{3}$ ENT Resident, ENT Department, Hospital Italiano de Buenos \\ Aires, Argentina
}

\author{
Correspondence: Carlos Santiago Ruggeri, Hospital Italiano \\ de Buenos Aires, Peron 4190, PCI I 99, Buenos Aires, Argentina, \\ Tel +54I |49590200, int 8368, \\ Email carlos.ruggari@hospitalitaliano.org.ar
}

Received: June 15, 2018 | Published: November 02, 2018

\section{Introduction}

Ecthyma gangrenosum is a necrotizing skin infection caused by gram-negative bacteria, mainly Pseudomonas aeruginosa (PA). It is associated with the presence of bacteremia due to PA, in immunocompromised patients. ${ }^{1}$ It is characterized by producing ulcers surrounded by an erythematous halo, and as it progresses, a black eschar is also added. ${ }^{2}$ A differential diagnosis should be made with acute invasive fungal rhinosinusitis, which also occurs in immunocompromised patients. The initial symptoms are usually not very specific, including fever, nasal obstruction and rhinorrhea. The aim of this report is to present the case of a male patient diagnosed with multiple myeloma who presented a facial Gangrenous Ecthyma due to Pseudomonas aeruginosa, with rhinosinusal onset.

\section{Case report}

A 60-year-old male patient presented with edema, congestion, and discharge and localized pain on the left eye, progressive and one week old. He had a history of multiple myeloma with immunoglobulin $\mathrm{G}$ kappa, diagnosed 4 months before the consultation, and in treatment with bortezomib (two complete cycles).

I was taking aspirin (100 mg/day), acyclovir (400 mg every 12 hours), trimethoprim sulfamethoxazole (160/800 mg tri-weekly) and dexamethasone (40 mg. trisemanal).

By Otorhinolaryngological and ophthalmologic examination, edema was diagnosed in the left side of the left side of the brain that was slightly extended to the contralateral sidewall, bilateral bipalpebral edema with inability to open the left eye with orbital edema and severe compromise of visual acuity, lesions with a blackish appearance at the epidermal level and purulent rhinorrhea. (Figure 1)

A nasal endoscopy with rigid $30^{\circ}$ optic was performed, and a pale mucosa was seen in the left nasal cavity with bloody discharge and free mucous fragments in the nasal cavity, apparently coming from the inferior turbinate. Mucosal fragments were sent for histopathological study by freezing, and samples were taken for blood culture. Computed tomography of the facial mass showed a thickening at the level of the facial teguments with predominance of the left, partial veiling of the maxillary sinus and left anteroposterior ethmoids, occupation of the nasal cavity and proptosis of the left eye, without evidence of intraorbital collection. Treatment was started with intravenous amphotericin B and empirical antibiotic therapy.

The histopathological study report was an acute inflammatory process with necrosis.

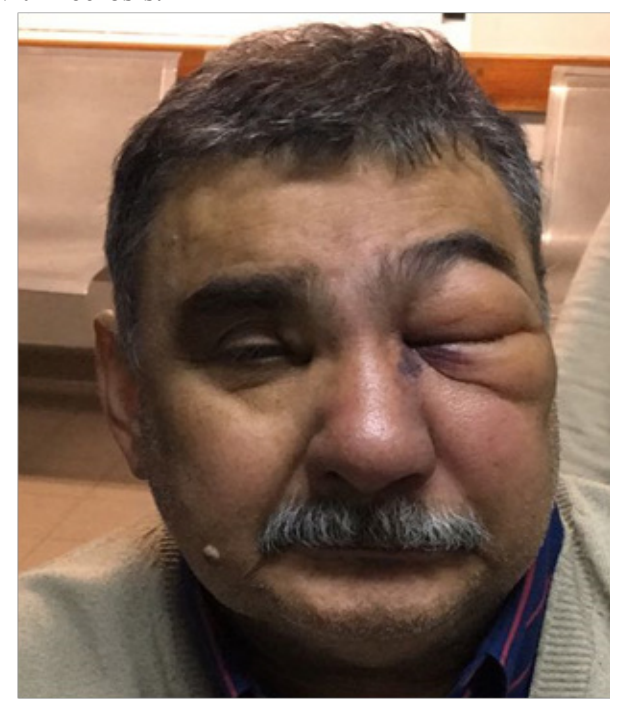

Figure I Patient during initial consultation.

A few hours after the initial consultation, a transnasal surgical approach with endoscopes was performed under general anesthesia, visualizing a pale nasal mucosa, which bled little. A partial turbinectomy, bilateral maxillary antrostomy and left anteroposterior ethmoidectomy were performed. The material was sent for anatomopathological study, for direct examination and bacteriological and mycological culture.

He was admitted to the intensive care unit. The disease progressed: palpebral edema increased and there was a complete loss of visual acuity in the left eye and a visible bulge in the right eye.

The pathological anatomy report was an acute inflammatory process with extensive necrosis and acute osteomyelitis. PAS and Grocott techniques were negative.

The patient continued to worsen, with extension of the black necrotic lesions at the facial level. Figure 2 


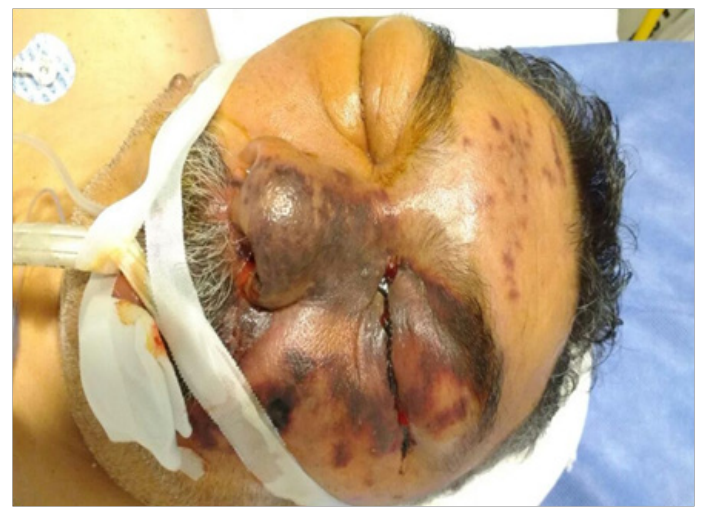

Figure 2 Progression of the lesions in the left side of the face and facial edema.

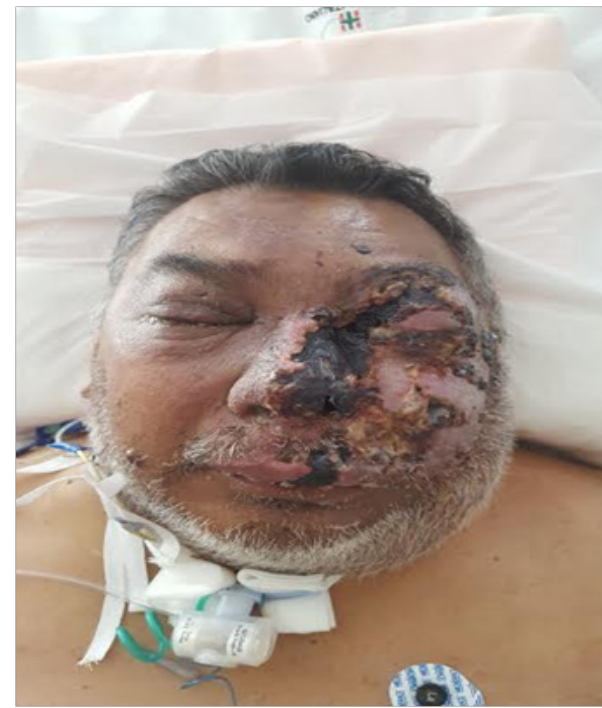

Figure 3 Extension of hemifacial necrotic lesions with severe involvement at the nasal and ocular levels.

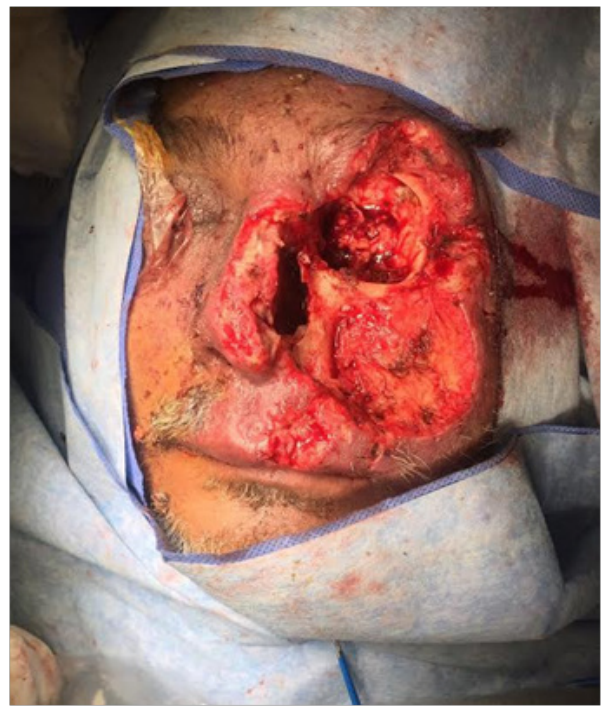

Figure 4 Exenteration and resection of facial necrotic areas.

In the initial blood culture, Pseudomonas aeruginosa sensitive to Amikacin and Colistin was diagnosed. A conjunctival swab revealed the presence of gram-negative bacilli and PCR was positive for
Herpes virus. The culture of the material obtained in the surgery also diagnosed Pseudomonas aeruginosa with the same sensitivity profile. Five days later, due to the progressive nature and poor response to treatment, a new rhinosinusal endoscopic surgery was performed to resect necrotic tissue, biopsy the facial skin and a tracheostomy was performed due to difficulties in extubating the patient. Gangrenosum ecthyma was diagnosed by Pseudomonas aeruginosa. Skin and mucosal biopsies detected necrosis of the superficial vessel walls and the presence of fibrin thrombi that occupied all the light of the same with isolated diffuse mixed inflammatory infiltrates and in the cultures continued to detect Pseudomonas aeruginosa.

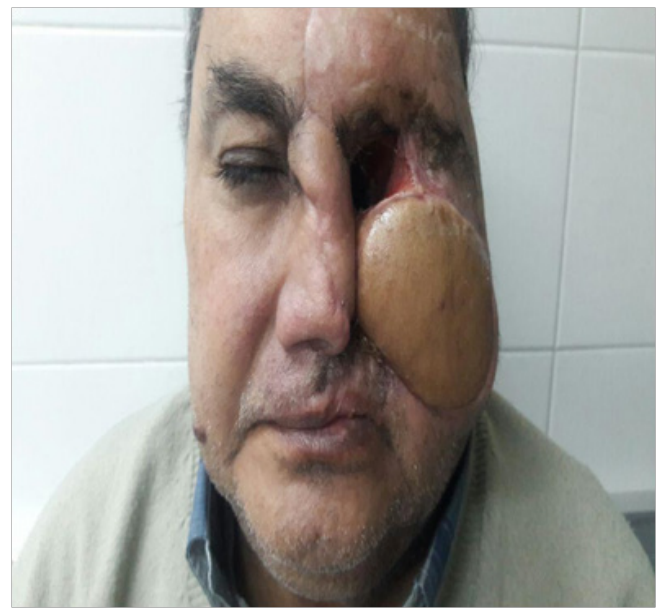

Figure 5 Actual picture of patient.

An arteriovenous angiotomography of the brain, orbit and neck was performed, which reported partial thrombosis of the left jugular vein and total left thoolinguofacial venous trunk as well as thrombosis of the right internal jugular vein. Thrombosis of the left pterygoid venous plexus and the ipsilateral retromandibular and anterior jugular veins was also observed.

Due to the progressive evolution of the hemifacial necrotic lesions (Figure 3), and the involvement of the orbital contents that produced blindness, 5 days later the left orbital exenteration was performed, and the resection of the necrotic facial tissue, until vital tissue was found. (Figure 4)

The patient evolved favorably, after completing the treatment with colistin and intravenous ampicillin for a total of 36 days.

After curing the infection, the nose was reconstructed with an Indian flap and the facial region with a pediculated muscle-cutaneous flap of the posterior trapezius Figure 5. The treatment with bortezomib was terminated, and multiple myeloma could be controlled.

\section{Discussion}

Ecthyma gangrenosum is a necrotizing skin infection caused by gram-negative bacteria, mainly Pseudomonas aeruginosa. It is usually associated with the presence of bacteremia by said bacteria, in immunocompromised patients. The most frequently affected sites are the gluteal, perineal region (57\%), and extremities (30\%). Only $6 \%$ of cases occur in the head and neck, and there is only one publication in the international literature with localization in the rhinosinusal mucosa. ${ }^{1}$ The gangrenous ecthyma is characterized by ulcerated lesions surrounded by an erythematous halo, as the ulcers progress, they become black eschar surrounded by erythema. ${ }^{2}$ The pathogenesis is unknown but there are several theories: the lesion is 
considered a seeding in the skin of Pseudomonas aeruginosa carried by blood in patients with bacteremia, another explanation in ecthyma gangrenosum without bacteremia, is that the initial necrotic ulcer is the entry point of the bacterium in the body, or it would represent an early form of infection. ${ }^{2}$ The only case published with localization in the rhinosinusal mucosa, has many similarities with the patient described in this work. The two patients had active oncohematological disease, a characteristic finding of Pseudomonas aeruginosa in the cultures of the biopsies and pathological anatomy was diagnosed thrombosis and extensive necrosis of the affected tissues. One of the most significant differences was that in the clinical case described in the previous study Pseudomonas bacteremia was not found, which is related to a better prognosis.

Patients who have the infection and have bacteremia have a mortality of $38 \%$ to $96 \%$, and in patients without bacteremia the mortality is $15 \%{ }^{2}$ The histopathological characteristic of gangrenous stigma is the invasion of the tunica media and the adventitia of the vascular walls without involvement of the intimal. ${ }^{3}$ Gram-negative bacteria are dispersed within the dermis, and there is a mixed inflammatory infiltrate and vascular proliferation that affects the subcutaneous tissue. ${ }^{4,5}$

The treatment of ecthyma gangrenosum includes the appropriate use of systemic antibiotic therapy according to sensitivity through biopsy cultures and blood cultures and local debridement of the necrotic tissue until vital tissue is found. The differential diagnosis should be made with acute invasive mycosis, and mid-line $\mathrm{T}$ lymphomas. It is essential to implement the treatment quickly and quickly recognize the disease since early treatment can lead to a better prognosis for the patient.

\section{Conclusion}

The gangrenous ecthyma in an unusual disease caused mainly by Pseudomonas aeruginosa and is more frequent in immunosuppressed patients with active oncohematological disease.

The knowledge of this rare infection and the early and adequate treatment is directly related to a lower morbidity and better survival.

\section{Acknowledgements}

None.

\section{Conflict of interest}

We declare there is no financial interest or any conflict of interest.

\section{References}

1. Hekiert AM, Cohen MB, Montone KT, et al. Ecthyma gangrenosum mimicking acute invasive fungal sinusitis in an inmunocompromised patient. Ear Nose Throat J. 2010;89(6):262-267.

2. Song WK, Kim YC, Park HJ, et al. Ecthyma Gangrenosum without Bacteraemia in a Leukaemic Patient. Clin Exp Dermatol. 2001;26:39597.

3. Solowski NL, Yao FB, Agarwal A, et al. Ecthyma Gangrenosum: A Rare Cutaneous Manifestation of a Potentially Fatal Disease. Ann Otol Rhinol Laryngol. 2004;113(6):462-464.

4. Kim HJ, Grossniklaus HE, Wojno TH. Periorbital Ecthyma Gangrenosum: A Case Report and Review of the Literature. Ophthalmic Plast Reconstr Surg. 2014;30(5):e125-e128.

5. Kelley DJ. Ecthyma Gangrenosum of the Nasal Cavity. Otolaryngology Head and Neck Surgery. 2003;129(6):754-755. 